\section{International Classification of Functioning, Disability and Health} (WHO)

Helga Peter ${ }^{1}$ und Thomas Penzel ${ }^{2}$

${ }^{1}$ Marburg, Deutschland

${ }^{2}$ Interdisziplinäres Schlafmedizinisches Zentrum, Charité Universitätsmedizin Berlin, Berlin, Deutschland

\section{Synonyme}

ICF

\section{Definition}

Internationale Klassifikation der Funktionsfähigkeit, Behinderung und Gesundheit.

Siehe $\triangleright$ „Leistungs-, Schläfrigkeits- und Vigilanzmessung". 